\title{
Photodynamic and Tissue Tolerable Plasma Therapies as Alternatives to Antimicrobials to Control Pathogenic Biofilms
}

\author{
Marco Aurelio Paschoal • \\ Thereza Cristina Botelho Dantas • Simone Duarte
}

Published online: 11 January 2015

(C) Springer International Publishing AG 2015

\begin{abstract}
The need for novel antimicrobial techniques has become critical for a number of reasons, including the emergence of resistant strains because of excessive prescription and misuse of antibiotics. The mouth is colonized by a large number of microorganisms. When these microorganisms are organized as biofilms they can contribute to chronic diseases in the human population, such as dental caries, candidiasis, and periodontal disease. Hence, alternative antimicrobial approaches have risen to facilitate the treatment of these diseases. As novel therapies, photodynamic antimicrobial chemotherapy and tissue-tolerable plasma are promising methods that offer the possibility of microbial decontamination with decreased odds for the traditional side effects. Thus, the present review aims to offer an overview and future directions of these new approaches to control pathogenic oral biofilms.
\end{abstract}

Keywords Photochemotherapy · Photodynamic antimicrobial chemotherapy · Tissue-tolerable plasma . Microorganism $\cdot$ Biofilm

This article is part of the Topical Collection on Microbiology

\section{A. Paschoal}

Master Program in Dentistry, CEUMA University, São Luis, MA, Brazil

e-mail: marcobpaschoal@hotmail.com

T. C. B. Dantas

Dentistry Post-Graduation Program, Ceará Federal University, Fortaleza, CE, Brazil

e-mail: therezacfb@hotmail.com

T. C. B. Dantas $\cdot$ S. Duarte $(\bowtie)$

Department of Basic Science and Craniofacial Biology, NYU College of Dentistry, New York University, New York, NY, USA

e-mail: sduarte@nyu.edu

\section{Introduction}

The increased microbial resistance against commercially available antimicrobial drugs and substances have cooperated with the search for alternative treatments for the control of pathogenic biofilms involved with diseases that affect the body, including biofilm-dependent oral diseases $[1,2]$. Photodynamic antimicrobial chemotherapy (PACT) is an alternative therapy that involves the concomitant combination of photosensitizers (PS) under proper light activation, aiming to generate toxic species of oxygen resulting in microbial death, while still being safe for the mammalian cells [3]. Following the same path, another novel therapy with a similar mechanism of action is tissue-tolerable plasma (TTP), an ionized gas produced by subjecting one or more gases to an electric field, of either constant or alternating amplitude [4]. The advantages of PACT and TTP over other antibiotic applications are that they can be used for site-specific treatment. they provide almost instantaneous bacterial response, and there is less chance for the development of bacterial resistance and minimal side effects [1]. It is generally assumed that oxygen and nitrogen-based radicals are the most significant contributors to the sterilizing effects of PACT and TTP [5]. Hence, this review aims to provide an overview of (i) in vitro and in vivo studies of both PACT and TTP therapies to treat oral biofilms, and (ii) additional applications in dentistry, as well as to discuss their mechanisms of action and future directions for the field.

\section{Photodynamic Antimicrobial Chemotherapy in Dentistry}

Treatment using light and PS activated by light were mentioned in ancient times and were used to treat a wide variety of 
disorders or diseases [6]. Different nomenclatures are given to this process. PACT, when there is a combination of a chemical compound and light, or simply photodynamic inactivation (PDI) or photodynamic therapy (PDT), are the most common definitions [7, 8]. PACT/PDI/PDT can be defined as the administration of a non-toxic drug or dye known as PS, either systemically, locally, or topically, to a patient with a localized lesion (including some types of cancer), followed by illumination with visible light (e.g. light-emitting diode [LED] or lasers) at a proper wavelength, which will activate the PS and produce cytotoxic species and, consequently, the breakdown of cellular microstructures, infected tissue destruction, and cell death [9]. This approach is considered a minimally invasive procedure and can be repeated several times, with minimal chances of causing resistance due to different mechanisms of action and targets [1]. Furthermore, PACT/PDI ideally causes limited or no damage to the host tissues [10]. A substantial number of studies state that the photodynamic approach can also be used to kill microorganisms, and is considered a promising alternative to treating oral biofilms involved in several major oral diseases such as candidiasis [11, 12], dental caries [13], and periodontal disease [14]. In recent years, PACT/PDI has been proposed as an alternative treatment for localized bacterial infections [15].

A PACT/PDI/PDT regimen can be beneficial for the elimination of microorganisms during systematic therapies of biofilm-dependent diseases, and is able to inactivate some of the known antibiotic-resistant microbial pathogens [15]. Therefore, this approach would be beneficial for the treatment of biofilm-dependent oral diseases, considering the high accumulation of bacteria in the oral environment and the easy access to the site of the disease. This alternative therapy is already known to be effective for some localized microbial infections, such as oral candidiasis [11, 12], chronic wounds, dental caries [13], periodontal disease [14], and treatment of endodontic lesions [16].

The mechanism by which microbial cells die when exposed to PACT-generated reactive oxygen species (ROS) has been the subject of intense investigations. Some hypotheses are that after PS activation by light in a proper wavelength (corresponding to its absorption window), there is a transfer of energy from the activated PS and the available oxygen gives rise to the formation of highly reactive oxygen species, such as singlet oxygen and free radicals that oxidize biological substrates [17]. Another hypothesis is that after activation, PS can initiate a photochemical type I or II reaction $[18,19]$. For the type I reaction, the activated PS reacts by transferring either an electron or hydrogen to an oxygen molecule or other adjacent molecule to form an anion or cation radical, respectively $[18,19]$. These radicals are likely to react with molecular oxygen, resulting in ROS production $[18,19]$. Examples of ROS include superoxide anion $\left(\mathrm{O}_{2}{ }^{-}\right)$, hydroxyl radical $(\mathrm{OH})$, hydrogen peroxide $\left(\mathrm{H}_{2} \mathrm{O}_{2}\right)$, and singlet oxygen $\left({ }^{1} \mathrm{O}^{2}\right)$. The type II reaction is an energy transfer that occurs at the ground state of molecular oxygen and allows the production of singlet oxygen which is capable of reacting with cellular components, generating membrane permeability, irreversible damage, and microbial death [19, 20]. Enzymes such as superoxide dismutase, catalase, peroxidase, and a number of secondary scavengers are able to control the levels of oxidative species [16]. In addition, critical sites of action for singlet oxygen in PACT/PDI/ PDT include mitochondria, DNA, and lipid membranes [9]. As established by the literature, radiation and chemotherapy tend to damage the DNA and lead to apoptosis via cell-cycle checkpoints, growth arrest, and p53 activation $[21,22]$. Alternatively, PACT/PDI/PDT tends to act via acute stress response involving mitochondrial damage, cytochrome $\mathrm{C}$ release, and formation of an apoptosome caspase $[21,22]$. Acute challenges can affect the expression of proteins, especially transcription factors, which regulate the transcription of specific genes causing cell deregulation followed by cell death. Another possible mechanism of action is related to transcription factors; synthesis and expression of proteins are often induced by acute challenges and bind to certain regions on the DNA, resulting in transcription of genes and, consequently, production of different proteins causing cell deregulation followed by cell death [23].

An ideal PS should have a low level of dark toxicity (e.g. in the absence of light does not present any damage effect), absorb light in the corresponding therapeutic window, null activity related to mutagenicity/carcinogenicity, and be water soluble and easily eliminated by the body [24, 25]. PS are derived from the tetrapyrrole aromatic nucleus found in many naturally occurring pigments such as hemecholophyll and bacteriochlorophyll [24]. Such examples are represented by porphyrins and chlorins, which were the first dyes studied [24]. Both groups present an absorption band between 630 and $690 \mathrm{~nm}$ that can be activated by red light sources [19].

Synthetic, non-naturally conjugated pyrrolic ring dyes, and a non-tetrapyrrolic (naturally occurring or synthetic dye) are represented by rose bengal/erythrosine, toluidine blue, and methylene blue dyes, respectively. Most of these dyes can be excited with wavelengths between 400 and $500 \mathrm{~nm}$, corresponding to light sources in the blue region $[26,27]$.

Different approaches and protocols have been used to determine the concentration of PS required to have the desired antimicrobial effect, which is established by the targeted microorganisms, their growth mode (planktonic or biofilm), and PS solubility [19, 28].

In general, PS are activated by complementary wavelengths. For example, phenotiazine dyes (e.g. toluidine blue and methylene blue) are classified at the blue broadband and 
are correctly activated by red lights at $600-660 \mathrm{~nm}$ that corresponds to complementary wavelenght $[29,30]$. On this same way, red dyes, such as rose bengal, erythrosine, Photogem/Photosan can be irrdiated with wavelenghts between 400-500 nm that corresponds to the blue light spectrum $[31,32]$.

The literature presents three main categories of clinical PACT light sources: LEDs, lasers, and halogen lamps [33•]. Lasers demonstrate some advantages, such as monochromaticity, high efficiency of light delivery without substantial power loss, and coupling of single optical fibers, but their costs are usually high when compared with other light sources [33•]. On the other hand, LEDs are most suitable for PACT and are easier to use presenting low costs when compared with lasers [34]. Also, in terms of wavelengths, LEDs and halogen lamps are not as specific as lasers, therefore LEDs can be used for several PS with similar activation peaks [34]. Hence, the use of halogen lamps and blue LEDs that present lower potencies and lower tissue penetration are desired.

In the dental field, the usual PACT applications are related to accessible areas where light does not need to penetrate very deep (such as complementary to caries removal, caries prevention, herpes simplex, candidiasis, and periodontal disease) [35]. Although the current studies report on the success of the use of the aforementioned PS/lights, further studies are necessary to gain a better understanding of PACT mechanisms of action and toxicity.

The oral cavity sustains more than 700 species of microorganisms, few of which participate in the pathogenesis of various oral diseases, such as dental caries and candidiasis $[35,36]$. The main challenge regarding the treatment of these diseases resides in antimicrobial resistance, reoccurrence (treatment fail), interactions with other medical regimens, organ toxicity, staining potential, taste alteration, and compliance of the patients to achieve therapy success [36].

The efficacy of PACT for different pathogens is dependent on the type and concentration of the PS, as well as on the interaction of the PS with the light source [33•]. In the biofilm, the presence of an extracellular matrix allows stability and structural integrity, possibly limiting the diffusion of substances throughout the biofilm, and protecting the bacteria from inimical influences of antimicrobials and other environmental challenges [37]. Recent studies related to PACT application over Candida albicans [38] and Streptococcus mutans [39] using methylene blue/red LED (630 nm) and erythrosine/ laser $(532 \mathrm{~nm})$, respectively, achieved a high photokilling rate. Dovigo et al. [40] reported the inactivation of Candida $s p$. biofilms formed by clinical isolate strains after the biofilms were exposed to Photodithazine ${ }^{\circledR}$. Nastri et al. [41] state that the association of toluidine blue and diode laser light at $830 \mathrm{~nm}$ was effective against bacterial strains involved in periodontal disease, even though they were organized in biofilms, indicating it can be a coadjuvant method for the treatment of periodontal pockets. Araujo et al. [42] reported that curcumin in association with blue light was effective on the decontamination of dentine carious lesions contaminated with S. mutans and Lactobacillus spp. biofilms. However, possibly because of the limitation of light penetration and drug diffusion, microorganisms were less affected in the biofilm phase than the planktonic counterparts (references). Moreover, Lee et al. [43] were able to achieve significant cell death using erythrosine as a photosensitizing agent and a dental halogen light on S. mutans biofilm. Nevertheless, for dental use, several aspects, including energy, light irradiation times, and distance to the targeted area, must be carefully analyzed, aiming at tissue-tolerable temperature in the dentin, pulp, and other oral tissues.

\section{Tissue-Tolerable Plasma in Dentistry}

Plasma is an electrical discharge produced by subjecting one or more gases to an electrical field, either as a constant or alternating amplitude [4]. The term 'plasma' comes from Greek language and was introduced by Irving Lanmuir, an American chemist and physicist, in 1928. There are three main states of matter — solid, liquid, and gas. When a gas receives more energy, particles of the gas collide with each other. As a result, electrons and ions are produced and the gas becomes charged. This state of matter is called 'plasma', which is known as the fourth state of matter [44].

Plasma has several appealing features, including the ability to reach the gas phase without the need for elevated temperature $[45,46]$. The electrons then collide with the gas, and enhance the level of dissociation, excitation, and ionization. The ions and neutrons continue reasonably cold, therefore TTP does not cause thermal damage to the surfaces $[47,48]$. Bare hands may touch a TTP device without causing any heating, electrical shock, or other painful sensation [49]. Although TTP is not as well established as PACT, these characteristics open up the possibility of using plasmas for the treatment of heat-sensitive materials, including biological matter such as cells and tissues.

Plasma-based dental applications have attracted much attention in dental biomaterial modification [50, 51], tooth whitening [52, 53], dental material adhesion [54], and dental caries [55], and as an effective anti-oral biofilm agent [56, 57].

TTP may improve disinfection performance because this approach can kill several microorganisms, including bacteria and viruses [55, 58-63].

Scanning electron microscopy (SEM) was used to study how TTP exposure can impact Escherichia coli cell morphology [64]. The results show that $E$. coli cells presented severe morphological changes, including lysis (splitting of cells), which was attributed to one of two processes: membrane 
rupture caused by the charge build-up on the cells, or chemical attack by radicals such as $\mathrm{O}_{2}^{-}$and/or $\mathrm{OH}$. Microorganisms exposed to plasma are also exposed to an intense bombardment by these short-living reactive species, with a higher oxidation potential, probably provoking surface levels that the living cell cannot repair sufficiently quickly. In this sense, we can say that TTP destroys microorganisms by disrupting the cell wall using these highly reactive free radicals, without the use of heat, chemicals, or pressure [65]. This may explain why cells are, in many cases, destroyed very quickly [66].

Sterilization by TTP usually depends on the synergism of a few bacterial agents: energetic photons in the ultraviolet (UV) B and UVC range; reactive species, including oxygen free radicals; and charged species such as electrons and ions. Their relevance is dependent on the type of plasma source and the sample preparation, in addition to how the UV photons get absorbed in atmospheric air [67]. The main plasma sterilization mechanisms are etching, oxidation, and UV radiation. Thus, bacterial size and structure may affect plasma effectiveness in bacterial deactivation. Yang et al. [68] reported the bactericidal effect of TTP on S. mutans and L. acidophilus, which are major pathogens in dental caries. The results indicated that TTP treatment was very effective in the disinfection of oral bacteria. They found that $L$. acidophilus killing needed a longer TTP exposure when compared with $S$. mutans. One of the hypotheses is that L. acidophilus presents a larger cell $(\cong 1 \mu \mathrm{m} \times 3 \mu \mathrm{m})$, which could mean higher plasma tolerance when compared with the smaller $S$. mutans $(\cong 1 \mu \mathrm{m}$ in diameter) [68]. To accomplish the same killing intensity, up to $5 \mathrm{~min}$ of TTP exposure was necessary to deactivate $L$. acidophilus, which is contrary to the $10 \mathrm{~s}$ of TTP exposure needed to kill S. mutans.

In the same study [68], free-floating planktonic $S$. mutans and $L$. acidophilus were exposed to argon plasma for different periods of time. Leakage of intracellular proteins and nucleic acid related to bacterial killing were accessed by a UV-visible spectrometer. The absorbance peak intensity was the highest amount of intracellular proteins and DNAs. According to the findings, a very short plasma exposure of only $1 \mathrm{~s}$ significantly increased the killing of both bacteria, which was demonstrated by the dramatic leakage of the intracellular proteins and DNAs, which usually occurs when the cell membranes are damaged.

Lu et al. [49] showed that TTP could efficiently kill Enterococcus faecalis grown in biofilms, one of the main types of bacterium causing failure of root canal treatment, in several minutes. Pan et al. [69] also investigated the in vitro feasibility of using cold plasma against $E$. faecalis in infected root canals, and observed a significant decrease in the number of colonyforming units, after a prolonged exposure time of 8-10 min. Yamazaki et al. [70•] carried out experiments to evaluate the sterilizing effects of the low-frequency atmospheric pressure plasma on oral pathogenic microorganisms (S. mutans, $C$. albicans and E. faecalis) and to determine its potential for clinical application. The major findings were that the plasma was effective in sterilizing all three oral pathogenic microorganisms in a short exposure time.

Furthermore, the highest concentration of plasma energy occurs 5-6 $\mathrm{mm}$ beyond the plasma needle [71]. The plasma needle produces bactericidal agents locally and no excess of radicals remained at the end of the treatment period [71].

Sladek et al. [55] evaluated the antimicrobial activity of TTP against biofilm cultures of a key cariogenic bacterium, S. mutans, comparing TTP with $0.12 \%$ chlorhexidine mouth rinse. The authors suggested that TTP may benefit caries treatment not only because of its antimicrobial properties but also because TTP is a gas that acts in a non-contact way, which could implicate that it can diffuse toward the surface, allowing the treatment of surface irregularities, including cracks and fissures. Under the conditions of this study, TTP presented higher inhibitory effects against $S$. mutans biofilm when compared with $0.12 \%$ chlorhexidine. Moreover, the effectiveness of TTP is also dependent on the type of bacteria, culture medium, and input power of plasma.

Furthermore, considering dental material adhesion is one of the main concerns in dentistry, TTP has also been used to modify the surface of polymers and to deposit an inert protective layer on it (reference). The plasma processes can increase wettability, biocompatibility, and durability without influencing bulk material properties (reference). This feature of plasma may have great potential for dental application [72••].

Recently, there has been increasing interests on the effects of TTP. Since TTP has a bactericidal effect on oral pathogens, although it could not replace rotary instruments, it is expected that TTP will play an important role in the prevention and treatment of dental caries. Likewise, TTP is a potential method for disinfection of root canals and for the treatment of other infectious diseases of the oral cavity. There have been attempts to apply plasma technology in the various fields of dentistry. Although many studies are still in the early stages, the potential value of plasma for dental applications has been demonstrated $[44,57,60]$. In addition to safety concerns, the fundamental principles of how TTP interferes with tissues, cells, and the entire living body must be investigated. To enlarge the scope of plasma applications and place relevant research to practical use, interdisciplinary research with participation of dental professionals is required.

\section{Conclusions and Future Directions}

Although both PACT and TTP therapies are very promising, they are still not ready to replace classic therapy for treatment of oral diseases. However, these alternative approaches may improve, accelerate, and lower the cost of prevention and treatment of these diseases. Further studies should take into 
account aspects such as concentration of dye and highpotency light sources (important factors to be considered for PACT), as well as optimizing the exposure time and substance used to generate the TTP stream to further facilitate the elimination of oral biofilm bacteria.

\section{Compliance with Ethics Guidelines}

Conflict of Interest Dr. Marco Aurelio Paschoal declares no potential conflicts of interest relevant to this article. He has received a grant and support for research development from Fundação de Amparo à Pesquisa e ao Desenvolvimento Científico e Tecnológico do Estado do Maranhão (FAPEMA).

Dr. Thereza Dantas declares no potential conflicts of interest relevant to this article. She has received a grant and support from Coordenação de Aperfeiçoamento de Pessoal de Nível Superior (CAPES) for research development.

Dr. Simone Duarte declares no potential conflicts of interest relevant to this article. She has received a grant from the International Association for Dental Research (IADR).

Human and Animal Rights and Informed Consent This article does not contain any studies with human or animal subjects performed by any of the authors.

\section{References}

Papers of particular interest, published recently, have been highlighted as:

- Of importance

•- Of major importance

1. Anderson GG, O'Toole GA. Innate and induced resistance mechanisms of bacterial biofilms. Curr Top Microbiol Immunol. 2008;322:85-105.

2. Fux CA et al. Survival strategies of infectious biofilms. Trends Microbiol. 2005;13(1):34- 40.

3. Konan YN, Gurny R, Allemann E. State of the art in the delivery of photosensitizers for photodynamic therapy. J Photochem Photobiol B. 2002;66(2):89-106.

4. Moisan M et al. Low-temperature sterilization using gas plasmas: a review of the experiments and an analysis of the inactivation mechanisms. Int J Pharm. 2001;226(1-2):1-21.

5. Maisch T. Anti-microbial photodynamic therapy: useful in the future? Lasers Med Sci. 2007;22(2):83-91.

6. Allison RR, Mang TS, Wilson BD. Photodynamic therapy for the treatment of nonmelanomatous cutaneous malignancies. Semin Cutan Med Surg. 1998;17(2):153-63.

7. Hamblin MR, Hasan T. Photodynamic therapy: a new antimicrobial approach to infectious disease? Photochem Photobiol Sci. 2004;3(5):436-50.

8. Komerik N, MacRobert AJ. Photodynamic therapy as an alternative antimicrobial modality for oral infections. J Environ Pathol Toxicol Oncol. 2006;25(1-2):487-504.

9. Konopka K, Goslinski T. Photodynamic therapy in dentistry. J Dent Res. 2007;86(8):694-707.

10. Meisel P, Kocher T. Photodynamic therapy for periodontal diseases: state of the art. J Photochem Photobiol B. 2005;79(2):159-70.

11. Calzavara-Pinton $\mathrm{P}$ et al. Photodynamic antifungal chemotherapy. Photochem Photobiol. 2012;88(3):512-22.
12. Souza RC et al. Comparison of the photodynamic fungicidal efficacy of methylene blue, toluidine blue, malachite green and lowpower laser irradiation alone against Candida albicans. Lasers Med Sci. 2010;25(3):385-9.

13. Teixeira AH et al. Effect of photodynamic antimicrobial chemotherapy on in vitro and in situ biofilms. Caries Res. 2012;46(6): 549-54.

14. Goulart Rde $\mathrm{C}$ et al. Photodynamic therapy in planktonic and biofilm cultures of Aggregatibacter actinomycetemcomitans. Photomed Laser Surg. 2010;28 Suppl 1:S53-60.

15. Tseng $\mathrm{SP}$ et al. Toluidine blue $\mathrm{O}$ photodynamic inactivation on multidrug-resistant Pseudomonas aeruginosa. Lasers Surg Med. 2009;41(5):391-7.

16. Souza LC et al. Photodynamic therapy with two different photosensitizers as a supplement to instrumentation/irrigation procedures in promoting intracanal reduction of Enterococcus faecalis. J Endod. 2010;36(2):292-6.

17. Tuite EM, Kelly JM. Photochemical interactions of methylene blue and analogues with DNA and other biological substrates. J Photochem Photobiol B. 1993;21(2-3):103-24.

18. Dougherty TJ et al. Photodynamic therapy. J Natl Cancer Inst. 1998;90(12):889-905.

19. Castano AP, Demidova TN, Hamblin MR. Mechanisms in photodynamic therapy: part one-photosensitizers, photochemistry and cellular localization. Photodiagnosis Photodyn Ther. 2004;1(4): 279-93.

20. Wainwright M. Photodynamic antimicrobial chemotherapy (PACT). J Antimicrob Chemother. 1998;42(1):13-28.

21. Boulikas T, Vougiouka M. Cisplatin and platinum drugs at the molecular level. Oncol Rep. 2003;10(6):1663-82.

22. Pruschy $\mathrm{M}$ et al. Key targets for the execution of radiation-induced tumor cell apoptosis: the role of p53 and caspases. Int J Radiat Oncol Biol Phys. 2001;49(2):561-7.

23. Villard J. Transcription regulation and human diseases. Swiss Med Wkly. 2004;134(39-40):571-9.

24. Detty MR, Gibson SL, Wagner SJ. Current clinical and preclinical photosensitizers for use in photodynamic therapy. J Med Chem. 2004;47(16):3897-915.

25. Allison RR et al. Photosensitizers in clinical PDT. Photodiagnosis Photodyn Ther. 2004;1(1):27-42.

26. Stockert JC et al. Photodynamic damage to HeLa cell microtubules induced by thiazine dyes. Cancer Chemother Pharmacol. 1996;39(1-2):167-9.

27. Bottiroli $\mathrm{G}$ et al. Enzyme-assisted cell photosensitization: a proposal for an efficient approach to tumor therapy and diagnosis. The rose bengal fluorogenic substrate. Photochem Photobiol. 1997;66(3): 374-83.

28. Paschoal MA et al. Streptococcus mutans photoinactivation by combination of short exposure of a broad-spectrum visible light and low concentrations of photosensitizers. Photomed Laser Surg. 2014;32(3): 175-80.

29. Wood $\mathrm{S}$ et al. Erythrosine is a potential photosensitizer for the photodynamic therapy of oral plaque biofilms. J Antimicrob Chemother. 2006;57(4):680-4.

30. Vahabi $\mathrm{S}$ et al. The effect of antimicrobial photodynamic therapy with radachlorin and toluidine blue on streptococcus mutans: an in vitro study. J Dent (Tehran). 2011;8(2):48-54.

31. Paulino TP et al. Use of hand held photopolymerizer to photoinactivate Streptococcus mutans. Arch Oral Biol. 2005;50(3):353-9.

32. Bolean $\mathrm{M}$ et al. Photodynamic therapy with rose bengal induces GroEL expression in Streptococcus mutans. Photomed Laser Surg. 2010;28 Suppl 1:S79-84.

33. Nagata JY et al. Antibacterial photodynamic therapy for dental caries: evaluation of the photosensitizers used and light source properties. Photodiagnosis Photodyn Ther. 2012;9(2):122- 
31. This manuscript provides a current review of photodynamic therapy components aplied to dental caries treatment.

34. Wilson BC, Patterson MS. The physics, biophysics and technology of photodynamic therapy. Phys Med Biol. 2008;53(9):R61-R109.

35. Zanin IC et al. Susceptibility of Streptococcus mutans biofilms to photodynamic therapy: an in vitro study. J Antimicrob Chemother. 2005;56(2):324-30.

36. Rezusta A et al. In vitro fungicidal photodynamic effect of hypericin on Candida species. Photochem Photobiol. 2012;88(3): 613-9.

37. Paes Leme AF et al. The role of sucrose in cariogenic dental biofilm formation: new insight. J Dent Res. 2006;85(10):878-87.

38. de Souza SC et al. Photosensitization of different Candida species by low power laser light. J Photochem Photobiol B. 2006;83(1):34-8.

39. Ishiyama $\mathrm{K}$ et al. Bactericidal action of photogenerated singlet oxygen from photosensitizers used in plaque disclosing agents. PLoS ONE. 2012;7(5):e37871.

40. Dovigo LN et al. Photodynamic inactivation of clinical isolates of Candida using Photodithazine ${ }^{\circledR}$. Biofouling. 2013;29(9):1057-67.

41. Nastri L et al. Effects of toluidine blue-mediated photodynamic therapy on periopathogens and periodontal biofilm: in vitro evaluation. Int J Immunopathol Pharmacol. 2010;23(4):1125-32.

42. Araujo NC et al. Photodynamic effects of curcumin against cariogenic pathogens. Photomed Laser Surg. 2012;30(7):393-9.

43. Lee YH et al. The photodynamic therapy on Streptococcus mutans biofilms using erythrosine and dental halogen curing unit. Int J Oral Sci. 2012;4(4):196-201

44. Kim JH et al. Plasma in dentistry: a review of basic concepts and applications in dentistry. Acta Odontol Scand. 2014;72(1):1-12.

45. Kunhardt EE. Generation of large-volume, atmospheric-pressure, nonequilibrium plasmas. IEEE Trans Plasma Sci. 2000;28(1):189-200.

46. Kogelschatz U. Filamentary, patterned, and diffuse barrier discharges. IEEE Trans Plasma Sci. 2002;30(4):1400-8.

47. Laroussi M, Lu X. Room-temperature atmospheric pressure plasma plume for biomedical applications. Appl Phys Lett. 2005;87(11).

48. Stoffels E et al. Plasma needle: a non-destructive atmospheric plasma source for fine surface treatment of (bio)materials. Plasma Sources Sci Technol. 2002;11(4):383-8.

49. Lu XP et al. An RC plasma device for sterilization of root canal of teeth. IEEE Trans Plasma Sci. 2009;37(5):668-73.

50. Silva NRFA et al. Surface characterization of Ti and Y-TZP following non-thermal plasma exposure. J Biomed Mater Res B Appl Biomater. 2011;99B(1):199-206.

51. Dantas MCC et al. Comparison between the effect of plasma and chemical treatments on fiber post surface. J Endod. 2012;38(2):215-8.

52. Pan $\mathrm{J}$ et al. A novel method of tooth whitening using cold plasma microjet driven by direct current in atmospheric-pressure air. IEEE Trans Plasma Sci. 2010;38(11):3143-51.

53. Sun $\mathrm{P}$ et al. Tooth whitening with hydrogen peroxide assisted by a direct-current cold atmospheric-pressure air plasma microjet. IEEE Trans Plasma Sci. 2010;38(8):1892-6.

54. Cokeliler D et al. Modification of glass fibers to improve reinforcement: a plasma polymerization technique. Dent Mater. 2007;23(3): 335-42.

55. Sladek REJ et al. Treatment of Streptococcus mutans biofilms with a nonthermal atmospheric plasma. Lett Appl Microbiol. 2007;45(3):318-23.
56. $\mathrm{Du} \mathrm{TF}$ et al. Evaluation of antibacterial effects by atmospheric pressure nonequilibrium plasmas against Enterococcus faecalis biofilms in vitro. J Endod. 2012;38(4):545-9.

57. Koban I et al. Antimicrobial efficacy of non-thermal plasma in comparison to chlorhexidine against dental biofilms on titanium discs in vitro: proof of principle experiment. J Clin Periodontol. 2011;38(10):956-65.

58. Kuo SP et al. A microwave-augmented plasma torch module. Plasma Sources Sci Technol. 2006;15(2):266-75.

59. Tang YZ et al. Sublethal and killing effects of atmospheric-pressure, nonthermal plasma on eukaryotic microalgae in aqueous media. Plasma Process Polym. 2008;5(6):552-8.

60. Goree J et al. Killing of S-mutans bacteria using a plasma needle at atmospheric pressure. IEEE Trans Plasma Sci. 2006;34(4):131724.

61. Sladek REJ et al. Plasma treatment of dental cavities: a feasibility study. IEEE Trans Plasma Sci. 2004;32(4):1540-3.

62. Stoffels E et al. Plasma needle for in vivo medical treatment: recent developments and perspectives. Plasma Sources Sci Technol. 2006;15(4):S169-80.

63. Sladek REJ, et al. Investigation of possibilities of plasma treatment for dental caries. Ppc-2003: 14th IEEE International Pulsed Power Conference, 2003. Digest of Technical Papers. Vols 1 and 2:11091111.

64. Laroussi M. Nonthermal decontamination of biological media by atmospheric-pressure plasmas: review, analysis, and prospects. IEEE Trans Plasma Sci. 2002;30(4):1409-15.

65. Laroussi M. Sterilization of contaminated matter with an atmospheric pressure plasma. IEEE Trans Plasma Sci. 1996;24(3): 1188-91.

66. Moreau M, Orange N, Feuilloley MGJ. Non-thermal plasma technologies: new tools for bio-decontamination. Biotechnol Adv. 2008;26(6):610-7.

67. Vleugels $\mathrm{M}$ et al. Atmospheric plasma inactivation of biofilmforming bacteria for food safety control. IEEE Trans Plasma Sci. 2005;33(2):824-8.

68. Yang B et al. Oral bacterial deactivation using a low-temperature atmospheric argon plasma brush. J Dent. 2011;39(1):48-56.

69. Pan $\mathrm{J}$ et al. Cold plasma therapy of a tooth root canal infected with enterococcus faecalis biofilms in vitro. J Endod. 2013;39(1):10510.

70. Yamazaki $\mathrm{H}$ et al. Microbicidal activities of low frequency atmospheric pressure plasma jets on oral pathogens. Dent Mater J. 2011;30(3):384-91. This manuscript is important because it describes a new research prototype model suggesting the use of plasma in smalland difficult to reach environments.

71. Kim SJ, Chung TH, Bae SH. Striation and plasma bullet propagation in an atmospheric pressure plasma jet. Phys Plasmas. 2010;17(5).

72.• Kim JH et al. Plasma in dentistry: a review of basic concepts and applications in dentistry. Acta Odontol Scand. 2014;72(1):112. This manuscript is of major importance because it is the most recent review regarding the use of plasma in dentistry. The authors broadly describe the most important concepts to support the use of TTP against oral pathogens. 